\title{
Suspect a PE? Less may be more
}

\author{
Fiona Godlee editor, BMJ
}

This week sees the start of a new series in the $B M J$. As part of our Too Much Medicine campaign (www.bmj.com/too-muchmedicine), the series aims to highlight clinical conditions in which overdiagnosis exposes patients to unnecessary and potentially harmful care. The first article, by Renda Soylemez Wiener and colleagues, looks at pulmonary embolism (doi:10. 1136/bmj.f3368). The authors conclude that the advent and widespread uptake of high resolution CT pulmonary angiography has greatly increased the detection of small isolated pulmonary emboli that would have caused no trouble to the patient and did not need to be found.

This is an important claim. In reaching it the authors rely on clinical research and administrative data. Most crucially, they point to the substantial increase in incidence of pulmonary embolism after CT pulmonary angiography was introduced. This occurred with little change in the mortality and despite assertive campaigns to ensure systematic use of thromboprophylaxis.

If many of these detected emboli are not clinically important, one might expect asymptomatic emboli to show up as incidentalomas at autopsy and on CT scans performed for other reasons. And this is exactly what studies have shown, say the authors. What we don't have is definitive evidence that untreated patients would come to no harm, and we are left not knowing exactly which emboli can be safely ignored.

As the authors say, the idea that pulmonary embolism can be overdiagnosed will be new and counterintuitive for some clinicians. But they stress that harms of overdiagnosis are just as real as those of underdiagnosis. These include harms associated with the test itself (unnecessary exposure to nephrotoxic contrast and carcinogenic radiation) and the risks of major bleeding while on warfarin. The authors report that these risks are significantly greater than the risks of a recurrent thromboembolism in patients with small isolated pulmonary emboli.

So what's to be done? We need to know more about which small emboli require treatment and which can be left alone. But while we wait for this information, the authors suggest that clinicians should challenge prevailing assumptions about how to manage patients with suspected pulmonary embolism. Not all patients need imaging: validated algorithms such as the Wells score should be used to identify those at highest risk. Not all imaging needs to take the form of CT pulmonary angiography: VQ scanning and Doppler ultrasonography may be more appropriate in some patients. And not all emboli need treatment: if properly informed of the benefits and risks, stable patients with isolated subsegmental pulmonary emboli may choose to be monitored rather than anticoagulated. The safety of this approach is currently being evaluated in a prospective cohort study.

Other conditions that will be covered in the series include chronic kidney disease, dementia, attention-deficit/hyperactivity disorder, chronic obstructive pulmonary disease, depression, and thyroid cancer, and we welcome further suggestions from you. Our hope is to build up a picture of the pathognomonic features of an overdiagnosed condition, and the beginnings of a list appear in the linked editorial (doi:10.1136/bmj.f4247). Please add to the list via rapid responses, and if you are interested in this issue, join us in Dartmouth in September for the Preventing Overdiagnosis conference (www. preventingoverdiagnosis.net). 